\title{
ON THE CONVERGENCE OF DIFFERENCE SCHEMES \\ FOR A HEAT-CONDUCTION EQUATION WITH DISCONTINUOUS COEFFICIENTS*
}

\author{
A. A. Samarskil and I. V. Fryazinov \\ Moscow
}

(Received 14 May 1961)

THERE is an extensive literature on difference methods of solving equations of the parabolic type. A considerable proportion of the studies concerns equations with constant coefficients. In a number of papers (see, for example, [1]-[6]) the stability and convergence of schemes with continuous and sufficiently smooth coefficients are studied. For example, in [4] the convergence and stability in the mean are proved (see $\S 1$, Section 2), while uniform stability and convergence are proved in [1]-[3] and [6].

Extension to the case of discontinuous coefficients entails major difficulties, since in the vicinity of the discontinuity the difference scheme does not in general approximate the differential operator [7]. It is only possible to overcome these difficulties for the heat-conduction equation if use is made of the special a priori estimates obtained in [8].

In [7], [9] and [10] the concept is introduced of homogeneous difference schemes having one and the same computational algorithm at all points of the difference mesh for any coefficients of a differential equation drawn from some class of functions. In this paper we consider homogeneous through-computation schemes for solving linear equations of the parabolic type with discontinuous coefficients without separating out explicitly of the lines of discontinuity-more accurately, without any modifications of the scheme near the lines of discontinuity of the coefficients. Our attention is therefore mainly turned to the question of the convergence of through-computation schemes in the class of discontinuous coefficients.

This question was studied for a quasilinear equation in [11], where proof was given of the convergence of the scheme $\mathcal{P}_{h \tau}^{(1)}($ see $\S 1$, Section 3$)$ for the case of moving ("oblique") discontinuities of the heat-conduction coefficient, on the assumption that $h^{2} / \tau \rightarrow 0$ when $h \rightarrow 0$ and $\tau \rightarrow 0$ (See also [12]). In this paper this assumption is copied for the linear heat-conduction equation.

We shall briefly describe the contents of the present paper.

In $\S 1$ we introduce the initial family of homogeneous difference schemes $p_{h \tau}^{(\alpha)}$ and formulate the mixed difference problem.

* Zh. vych. mat. 1: No. 5, 806-824, 1961. 
In $\S 2$, with the aid of a priori estimates [8] and the maximum principle, a study is made of the stability of schemes with respect to the right-hand side using various norms.

Of especial interest is the case of the symmetrical six-point scheme

$$
\left(* * * \mid \begin{array}{l}
0.5 \\
* * * \\
0.5
\end{array}\right) \quad(\alpha=0.5)
$$

for which proof has been given of the stability in the mean with respect to initial data

$$
\|z\|_{2} \leqslant M\left\|z^{0}\right\|_{2}
$$

(see (10), $\S 2$, Section 2) and also of the stability with respect to the right-hand side $\Psi$ :

$$
\|z\|_{2} \leqslant M\|\tilde{\Psi}\|_{2} .
$$

These estimates are valid for the case when the lines of discontinuity of the coefficients are the straight lines $x=$ const. ("stationary discontinuities"), and the coefficient of heat conduction $k(x, t)$ and thermal capacity $c(x, t)$ satisfy with respect to $t$ the Lipschits condition.

For the case of an oblique discontinuity use is made of the a priori estimate [8] with respect to the norm $\left(z^{2 n}, 1\right)^{\ddagger n}$, where $n=1,2,3, \ldots$.

In $\S 3$ proof is given of the convergence of the family under consideration in the class of discontinuous coefficients, and estimates are given of the rate of convergence (order of accuracy) with respect to $h$ and $\tau$. It is shown that the scheme $\mathcal{P}_{h=}^{(\alpha)}$ with standard functionals

$$
A[\psi(s)]=\left[\int_{-1}^{0} \frac{\mathrm{d} s}{\psi(s)}\right]^{-1}, \quad D[\psi(s)]=F[\psi(s)]=R[\psi(s)]=\int_{-0.5}^{0.5} \psi(s) \mathrm{d} s
$$

possesses better accuracy than other schemes from the family under consideration [10].

\section{\$1. HOMOGENEOUS DIFFERENCE SCHEMES}

1. Initial problem. In the rectangle $\bar{\not}(0 \leqslant x \leqslant 1,0 \leqslant t \leqslant T)$ we shall find the function $u(x, t)$ satisfying the diferential equation

$$
\supset \boldsymbol{u}=L^{(\boldsymbol{k}, q, f)} u-c(x, t) \frac{\partial u}{\partial t}=0 \text { in the domain } \bar{\not}
$$

with additional conditions

where

$$
u(x, 0)=u_{0}(x), \quad u(0, t)=u_{1}(t), \quad u(1, t)=u_{2}(t)
$$

$$
L^{(k, q, f)} u=\frac{\partial}{\partial x}\left(k(x, t) \frac{\partial u}{\partial x}\right)-q(x, t) u+f(x, t)
$$


We shall assume that the coefficients of equation (1) are piecewise-continuous and satisfy the condition

$$
0<k_{*} \leqslant k(x, t) \leqslant k^{*}, \quad 0<c_{*} \leqslant c(x, t) \leqslant c^{*}, \quad 0 \leqslant q(x, t) \leqslant q^{*} \text { in } \bar{\not} .
$$

where $k_{*}, k^{*}, c_{*}, c^{*}$ and $q^{*}$ are constants.

If the coefficient $k(x, t)$ experiences a discontinuity on a finite number of curves $\Gamma_{v}, v=1,2, \ldots, v_{0}$ then on each of these curves the solution of equation (1) satisfies the continuity conditions

$$
\left.[u]\right|_{\Gamma_{v}}=0,\left.\left[k \frac{\partial u}{\partial x}\right]\right|_{\Gamma_{v}}=0, \quad v=1,2, \ldots, v_{0},
$$

where $\left.[u]\right|_{\Gamma_{\nu}}=u_{r}-u_{l}$ is the difference of the right- and left-hand limit values on the curve $\Gamma_{v}$. The problem defined by conditions (1)-(4) will henceforward be called problem (I).

The curves $\Gamma_{v}$, which are defined for the range $0 \leqslant t \leqslant T$ by the equations $x=\eta_{v}(t)$, will be relabelled so that $\eta_{v_{1}}(t)<\eta_{v_{2}}(t), v_{1} \leqslant v_{2}$; we assume, moreover, that the curves $\Gamma_{v}\left(v=0,1,2, \ldots, v_{0}, v_{0}+1 ; \eta_{0}(t)=0, \eta_{v_{0}+1}=1\right)$ are differentiable when $0 \leqslant t \leqslant T$ and do not intersect in pairs in $\overline{\bar{z}}$. We shall write

$$
\Delta_{v}=\left(\eta_{v}(t)<x<\eta_{v+1}(t), \quad 0<t \leqslant T\right), \quad \not=\sum_{v=0}^{v_{0}} \Delta_{v} .
$$

Proved in [13] is the theorem of the existence and uniqueness of a solution of problem (1)-(4) possessing in $\not$ the derivatives $\partial u / \partial t, \partial^{2} u / \partial x^{2}$, which in each of the domains $\Delta_{v}$ satisfy Hölder's conditions with respect to $x$ and $t$.

We shall henceforward assume the existence of the solution $u(x, t)$ of problem (I), without dealing with the question of the conditions which ensure the necessary degree of differentiability in $\not$ of the function $u(x, t)$.

2. Notations. For solving problem (I) by the method of finite differences we shall introduce into $\bar{\not} \bar{l}$ the difference mesh $\bar{\Omega}$, i.e., the set of points $\left(x_{i}, t_{j}\right)$ with coordinates $x_{i}=i h, i=0,1,2, \ldots, N, h=1 / N$ and $t_{j}=j \tau, j=0,1,2, \ldots, L, \tau=T / L$. We shall denote by $\Omega$ the set of internal points $\left(x_{i}, t_{j}\right)$ of the mesh $\bar{\Omega}$, for which $0<i<N$, $0<j \leqslant L$. The function $y_{i}^{j}$, defined on $\bar{\Omega}$ will be termed the mesh function.

For convenience we shall as a rule use the index-less notation and instead of $y_{i}^{J}$ will write simply $y$ or $y(x, t)$, putting

so that

$$
\begin{gathered}
y=y_{i}^{j}, \quad \stackrel{(+1)}{y}=y_{i+1}^{j}, \quad \stackrel{(-1)}{y}=y_{i-1}^{j}, \quad \check{y}=y_{i}^{j-1}, \quad \check{y}=y_{i}^{j-2}, \\
y_{\bar{x}}=\frac{y-\stackrel{(-1)}{y},}{h}, \quad y_{x}=\frac{\stackrel{(+1)}{y}-y}{h}, \quad y_{\tilde{x}}=0.5\left(y_{\bar{x}}+y_{x}\right), \quad y_{\bar{t}}=\frac{y-\check{y}}{\tau},
\end{gathered}
$$

$$
\begin{aligned}
\left(a y_{\bar{x}}\right)_{x} & =\frac{\stackrel{(+1)}{a} y_{x} \quad a y_{\bar{x}}}{h}=\frac{\stackrel{(+1)}{a}(\stackrel{+1)}{y}-y) a(y \stackrel{(-1)}{y})}{h^{2}} \\
& =\frac{1}{h^{2}}\left[a_{i+1}^{j}\left(y_{i+1}^{j}-y_{i}^{j}\right)-a_{i}^{j}\left(y_{i}^{j}-y_{i-1}^{j}\right)\right] .
\end{aligned}
$$


We shall employ the following sums and norms:

$$
\begin{gathered}
(y, z)=\sum_{i=1}^{N-1} y_{i} z_{i} h ; \quad[y, z)=\sum_{i=0}^{N-1} y_{i} z_{i} h ; \quad(y, z]=\sum_{i=1}^{N} y_{i} z_{i} h ; \quad[y, z]=\sum_{i=0}^{N} y_{i} z_{i} h, \\
\|y\|_{0}=\max _{0 \leqslant i \leqslant N}\left(y_{i}\right),\|y\|_{\sigma}=\left(|y|^{\circ}, 1\right)^{1 / \sigma} \quad(\sigma=1,2), \\
\|y\|_{3}=\|\tilde{y}\|_{2},\|y\|_{4} \quad\|\tilde{y}\|_{1}, \tilde{y}_{i}=\sum_{k=1}^{i} h y_{k},\|\tilde{y}\|_{\sigma}=\max _{0 \leqslant t \leqslant T}\|y\|_{\sigma}, \quad \sigma=0,1,2,3,4 .
\end{gathered}
$$

We shall also use the notation:

$$
\left\|y_{\bar{x}}\right\|_{0}=\max _{0<i \leqslant N}\left|y_{\bar{x}}\right|, \quad\left\|y_{\bar{x}}\right\|_{\sigma}=\left(\left|y_{\bar{x}}\right|^{\sigma}, \quad 1\right]^{1 / \sigma} \quad(\sigma=1,2) .
$$

It is easy to see that

$$
\|y\|_{4} \leqslant\|y\|_{3} \leqslant\|y\|_{1} \leqslant\|y\|_{2} \leqslant\|y\|_{0} .
$$

With the aid of these notations the first Green's difference formula may be written in the form

$$
\left(\left(a y_{\bar{x}}\right)_{x}, z\right)=-\left(a, y_{\bar{x}} z \bar{x}\right]+\left(a \cdot y_{\bar{x}} \cdot z\right)_{N}-\stackrel{(+1)}{\left(a \cdot y_{x} \cdot z\right)_{0} .}
$$

We shall designate by $\omega_{\tau}^{t}$ the set of points $\left(x, t^{\prime}\right)$ of the difference mesh, for which $t^{\prime}=\tau, 2 \tau, \ldots, t$; and by $\omega_{h}^{x}$ the set of points $\left(x^{\prime}, t\right)$ of the mesh for which $x^{\prime}=h, 2 h, \ldots, x ;$ thus for example

$$
\sum_{\omega_{\tau}^{t}}\|\psi\| \tau=\sum_{j^{\prime}=1}^{j}\left\|\psi^{j^{\prime}}\right\| \tau \quad \quad\left(t=t_{j}\right)
$$

3. Difference schemes. A study is made in [7] of the homogeneous difference schemes $L_{h}^{(k, q, f)}$ for the stationary heat-conduction equation $L^{(k, q, f)}=0$. We shall make use of these schemes.

We shall select as the initial family of difference schemes for problem (I) a family of six-point schemes:

$$
P_{h \tau}^{(\alpha)} y=\alpha L_{h}^{(k, q, f)} y+(1-\alpha) L_{h}^{(\check{k}, \check{q}, \check{f})} \check{y}-\rho^{(\alpha)} y_{\bar{t}}\left(\begin{array}{l|r}
* * * & \alpha \\
* * & 1-\alpha
\end{array}\right),
$$

where $0 \leqslant \alpha \leqslant 1$ is a numerical parameter, $q^{(\alpha)}=\alpha q+(1-\alpha) \check{q}$,

$$
L_{h}^{(k, q, f)} y=\left(a y_{x}\right)_{x}-d y+\Phi
$$

is a conservative three-point homogeneous scheme, the coefficients of which are defined by the standard functionals

$$
\begin{array}{ll}
A^{h}[\psi(s)], & -1 \leqslant s \leqslant 0, \\
D^{h}[\psi(s)], & -0.5 \leqslant s \leqslant 0.5, \\
F^{h}[\psi(s)], & -0.5 \leqslant s \leqslant 0.5,
\end{array}
$$

in accordance with the formulae

$$
a=A^{h}[k(x+s h, t)], \quad d=D^{h}[q(x+s h, t)], \quad \Phi=F^{h}[f(x+s h, t)] .
$$


The coefficient $\rho$ is computed with the aid of the standard functional $R^{h}[\psi(s)]$, $-0.5 \leqslant s \leqslant 0.5$ in accordance with the formula

$$
\rho=R^{h}[c(x+s h, t)] \text {. }
$$

The relationship of $a, \rho, d$ and $\Phi$ to the steps of the mesh $h$ and $\tau$ is not indicated.

The family of difference schemes $\mathcal{P}_{h \tau}^{(\alpha)}$ is defined, if a class of standard functionals $A^{h}, D^{h}, F^{h}$ and $R^{h}$ is prescribed.

In the general case, as is shown by the example of the accurate scheme formulated for the stationary heat-conduction equation in [9], the coefficients of the scheme can depend on all the coefficients of the differential equation. To facilitate our exposition we consider here schemes of the standard type, each coefficient of which depends only on one coefficient of the differential equation. However, as will be seen later, in the majority of cases the structure of the standard functionals does not figure explicitly.

We shall employ the concept, first introduced in [8], of the rank of the functional to characterize the class of standard functionals. Some functional $A^{h}[\psi]$, dependent on the parameter $h$, has a rank $r$ on the following conditions.

i. $A^{h}[\psi]$ has a differential of order $r$ with respect to $h$ :

$$
A^{h}[\psi]-A^{(0)}[\psi]+h A^{(1)}[\psi]+\ldots+h^{r} A^{(r)}[\psi]+h^{r} \rho(h),
$$

and each of the functionals $A^{(\sigma)}[\psi], \sigma=1,2, \ldots, r$ has a differential of order $r-\sigma$ with respect to the argument $\psi$ :

$$
A^{(\sigma \cdot}[\psi+\delta \cdot \varphi]=A^{(\sigma)}[\psi]+\delta \cdot A_{1}^{(\sigma)}[\psi, \varphi]+\ldots+\delta^{r-\sigma} A_{r-\sigma}^{(\sigma)}[\psi, \varphi]+\delta^{r-\delta} \rho(\delta) .
$$

(Here and henceforward $\rho(\varepsilon)$ denotes an expression tending uniformly to zero when $\varepsilon \rightarrow 0$.)

ii. The functional $A^{h}[\psi]$, and hence $A^{(\sigma)}[\psi]$ for all $\sigma=1,2, \ldots, r$ are homogeneous functionals of the first degree

$$
A^{h}[c \psi]==c A^{h}[\psi], \quad A^{(\sigma)}[c \psi]=c A^{(\sigma)}[\psi],
$$

where $c$ is a positive constant.

iii. The functionals $A^{h}[\psi]$ and $A^{(\sigma)}[\psi]$ are non-decreasing:

$$
A^{h}\left[\psi_{2}\right] \geqslant A^{h}\left[\psi_{1}\right], \text { if } \psi_{2} \geqslant \psi_{1},
$$

where $A^{h}[\psi]$ is a normalized functional:

$$
A^{h}[1]=1 \quad\left(A^{(0)}[1]=1, \quad A^{(0)}[1]=0 \text { when } \sigma \geqslant 1\right) .
$$

If $A^{h}[\psi]$ is a linear functional then all $A^{(\sigma)}[\psi]$ are also linear. Therefore condition ii and the requirement of differentiability with respect to $\psi$ are satisfied automatically.

We shall assume the following:

(1) the standard functionals $R^{h}[\psi], D^{h}[\psi]$ and $F^{h}[\psi]$ are linear, and have rank 2 and a second order of approximation, i.e.

$$
R^{h}[c(x+s h, t)]-c(x, t)=O\left(h^{2}\right)
$$

etc. : 
(2) the standard functional $A^{h}[\psi]$ has rank 3 and a second order of approximation:

$$
A^{h}[k(x+s h, t)]-k(\bar{x}, t)=O\left(h^{2}\right) . \quad \quad x=x-0.5 h .
$$

From the conditions $3^{0}$ and (3) it follows that

$$
0<k_{*} \leqslant a \leqslant k^{*}, \quad 0<c_{*} \leqslant p \leqslant c^{*}, \quad 0 \leqslant d \leqslant q^{*} .
$$

From condition (2) there follows especially the feasibility of the expression

$$
\begin{aligned}
a & =A^{h}[k(x+s h, t)]=k(\bar{x}, t)+h k^{\prime}(\bar{x}, t)\left(0 \cdot 5+A_{1}^{(0)}[s]\right)+ \\
& +h^{2}\left\{k^{\prime \prime}(\bar{x}, t)\left(\frac{1}{8}+0 \cdot 5 A_{1}^{(0)}[s]+0 \cdot 5 A_{1}^{(0)}\left[s^{2}\right]\right)+k^{\prime}(\bar{x}, t) A_{1}^{(1)}[s]+\right. \\
& \left.+\frac{\left(k^{\prime}(\bar{x}, t)\right)^{2}}{k(\bar{x}, t)} A_{2}^{(0)}[s]\right\}+O\left(h^{3}\right),
\end{aligned}
$$

where the accent denotes differentiation with respect to $x, \bar{x}=x-0.5 \mathrm{~h}$,

$$
A_{m}^{(\mathrm{o})}[1, \varphi(s)]=A_{m}^{(\mathrm{o})}[\varphi(s)] .
$$

From (1) it follows that

$$
\rho=R^{h}[c(x+s h, t)]=c(x, t)+h c^{\prime}(x, t) R^{(0)}[s]+O\left(h^{2}\right) ;
$$

etc.

The requirements of the second order of approximation

$$
\begin{gathered}
a=k(x-0.5 h, t)+O\left(h^{2}\right), \quad p=c(x, t)+O\left(h^{2}\right), \quad d=q(x, t)+O\left(h^{2}\right), \\
\Phi=f(x, t)+O\left(h^{2}\right)
\end{gathered}
$$

will be satisfied, if

$$
A_{1}^{(0)}[s]=-0.5, \quad R^{(0)}[s]=D^{(0)}[s]=F^{(0)}[s]=0 .
$$

For example, conditions (1) and (2) are satisfied by schemes in which

$$
\begin{aligned}
& a=k_{i-\frac{1}{k}}, \quad a=0.5\left(k_{i}+k_{i-1}\right), \quad a=\frac{2 k_{i} k_{i-1}}{k_{i}+k_{i-1}}, \\
& d=q_{i}, \quad d=0.5\left(q_{i+\xi}+q_{i-\mathfrak{k}}\right), \quad \Phi=f_{i}, \quad \Phi=0.5\left(f_{i-\mathfrak{t}}+f_{i+i}\right) . \\
& p=c_{i}, \quad \rho=0.5\left(c_{i+1}+c_{i-\xi}\right), \quad \text { where } \xi_{i \pm ł}=\xi\left(x_{i} \pm 0.5 h ; t\right) \text {. }
\end{aligned}
$$

From conditions (1) and (2) it follows in particular that the scheme $L_{h}^{(k, q, f)}$ and the scheme $\mathcal{P}_{h r}^{(\alpha)}$ have a second order of approximation with respect to $h$.

The question of the order of approximation of the scheme with respect to $\tau$ will be considered below. Conditions (1) and (2) and the parameter $\alpha$ determine the initial class of schemes.

If two schemes have an identical order of approximation (order of accuracy) then it is said [7], that these schemes are equivalent with respect to the order of approximation (accuracy).

If the standard functionals of a scheme do not depend on $h$, then such a scheme is termed canonical.

It is not difficult to show that any scheme $\mathscr{P}_{h \tau}^{(\alpha)}$, satisfying (1) and (2) is equi- 
valent to its canonical part, i.e., to a scheme with standard functionals $A^{(0)}, D^{(0)}$, $F^{(0)}, R^{(0)}$. It is therefore possible to confine ourselves to studying canonical schemes, to which group there belongs, in particular, the scheme $(\Lambda)$ cited in the introduction.

4. Difference boundary-value problkm. We shall correspond to problem (1) in $\bar{д}$ the following difference problem (II):

$$
\left.\begin{array}{l}
\mathcal{P}_{h \tau}^{(\alpha)} y=0 \quad \text { on } \Omega, \\
y^{0}=u_{0}(x), \quad y_{0}=u_{1}(t), \quad y_{N}=u_{2}(t)
\end{array}\right\}
$$

The conditions of continuity (4) are not formulated.

The main quantity characterizing the quality of the difference scheme is $z=y-u$, where $y$ is the solution of problem (II) and $u$ the solution of the corresponding problem (I).

The mesh function $z$ obviously satisfies the equation

and the homogeneous conditions

$$
\bar{\supset}_{h \tau}^{(\alpha)} z=-\Psi \text { on } \Omega
$$

where

$$
z=0 \text { when } t=0, x=0, x=1,
$$

$$
\overline{\mathcal{P}}_{h \tau}^{(\alpha)} z=\alpha L_{h}^{(k, q)} z+(1-\alpha) L_{h}^{(\grave{k}, \check{q})} \check{z}-\rho^{(\alpha)} z_{\bar{t}},
$$

$\Psi=\mathcal{P}_{h \tau}^{(\alpha)} u-\alpha \mathcal{P}_{u}-(1-\alpha) \check{\mathcal{P}}_{\mathfrak{u}} \mathfrak{u}$ is the error of approximation of the scheme $\mathcal{P}_{h \tau}^{(\alpha)}$ in the class of solutions of equation (1).

We shall represent $\Psi^{\circ}$ in the form of a sum:

$$
\Psi=\alpha \varphi+(1-\alpha) \check{\varphi}+\alpha \chi+(1-\alpha) \check{\chi}+r,
$$

where

$$
\begin{aligned}
& \varphi=L_{h}^{(k, q, f)} u-L^{(k, q, f)} u, \quad \chi=(c-\rho) \frac{\partial u}{\partial t}, \\
& r=\alpha \rho\left(\frac{\partial u}{\partial t}-u_{\bar{t}}\right)+(1-\alpha) \check{\rho}\left(\frac{\partial \breve{u}}{\partial t}-u_{\bar{t}}\right),
\end{aligned}
$$

It is obvious from this that the order of approximation with respect to $h$ of the scheme $\mathscr{P}_{h \tau}^{(x)}$ is determined by the order of approximation of the scheme $L_{h}^{(k, q, f)}$ and the functional $R^{h}[\psi(s)]$.

Requirements (1) and (2) secure a second order of approximation with respect to $h$ for the scheme $\mathcal{P}_{h \tau}^{(\alpha)}$.

It is easy to see, further, that $r=O\left(\tau^{m_{\alpha}}\right)$, where $m_{\alpha}=1$ when $\alpha \neq \frac{1}{2}, m_{1}=2$ and hence

$$
\Psi=O\left(h^{2}\right)+O\left(\tau^{m_{\alpha}}\right) .
$$

Thus the scheme $\mathcal{P}_{h \mathrm{r}}^{(\alpha)}$ has a second order of approximation with respect to $h$ and an order $m_{\alpha}$ with respect to $\tau$.

Differentiability is here assumed to the necessary number of times, both of the coefficients $k, q, c$ and $f$ and also the function $u$. 
In the case of discontinuous coefficients it turns out that the scheme $\mathcal{P}_{h \tau}^{(\alpha)}$ does not approximate the operator $\mathcal{P} u$ near the line of discontinuity (this is dealt with in greater detail in $\S 3$ ).

The question of the convergence when $h \rightarrow 0$ and $\tau \rightarrow 0$ of the solution of the difference problem (II) (scheme $\mathcal{P}\left(\begin{array}{c}(\alpha) \\ h \tau\end{array}\right)$ to the solution of problem (I) has been reduced to study of the asymptotic behaviour when $h \rightarrow 0$ and $\tau \rightarrow 0$ of the solution of problem (III) and its dependence on the magnitude of the error of approximation $\Psi$. For proof of convergence we require $a$ priori estimates of the solution of problem (III).

In passing we shall examine the question of the stability of our scheme with respect to initial data and the right-hand side. Henceforward we shall denote constants independent of $h$ and $\tau$ by $M$, without, as a rule, indicating their structure.

\section{§ 2. ON THE STABILITY OF DIFFERENCE SCHEMES}

We shall consider the question of the stability of difference schemes.

In the case of constant (or $x$-dependent) coefficients for schemes $\mathcal{P}_{h \tau}^{(\alpha)}$ in [14] and [15] it is shown that the requirement of stability in the mean of the scheme when $\frac{1}{2} \leqslant \alpha \leqslant 1$ does not impose limitations on the ratio of the steps $\gamma=\tau / h^{2}$ or on $\delta=\tau / h$. In the case when $0 \leqslant \alpha<\frac{1}{2}$ the requirement of stability of the scheme leads to the condition

$$
\frac{\tau}{h^{2}} \leqslant \frac{c_{*}}{2(1-2 \alpha) k^{*}} .
$$

It is natural to expect analogous results in the case of time-dependent coefficients as well.

Stability in the mean for smooth coefficients, dependent on time, was examined earlier for somewhat different schemes when $\alpha=\frac{1}{2}$ in [4].

Stability question will be examined by the method of integral estimates, on the assumption that

$$
\left|a_{\bar{t}}\right|,\left|\rho_{\bar{t}}\right|
$$

are limited. This requirement does not exclude the possibility of stationary discontinuities. We shall also refer to uniform stability.

1. Principle of the maximum. We know [16] that for four-point schemes

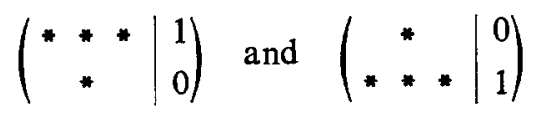

one can apply the principle of the maximum:

$$
\|z\|_{0} \leqslant M\left\{\left\|z^{0}\right\|_{0}+\sum_{\omega_{\tau}^{t}}\|\Psi\|_{0} \tau\right\}
$$

where

For the scheme

$$
\Psi=-\dddot{P}_{h \tau}^{(\alpha)} z, \quad z_{0}=z_{N}=0,\left.\quad z\right|_{t=0}=z^{0}
$$

$$
\left(* * * \mid \begin{array}{l|l}
* \\
* *
\end{array}\right)
$$


the preceding inequality takes place when $\gamma=\tau / h^{2} \leqslant c_{*} / 2 k^{*}$. Let $d \geqslant 0$. We shall show that estimate (5) is also satisfied for the scheme

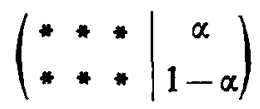

on the condition $\gamma \leqslant c_{*} / 2(1-\alpha) k^{*}$ (see also [5]).

We shall consider the six-point scheme $\mathcal{P}_{h \tau}^{(\alpha)}$ and write it in the form

$$
\begin{aligned}
& \alpha \tau\left[\left(a z_{\bar{x}}\right)_{x}-d z\right]-\rho^{(\alpha)} z=-\left\{\check { z } \left[\rho^{(\alpha)}-\gamma(1-\alpha)(\stackrel{(\breve{1} a)}{a}+\check{a})-\right.\right.
\end{aligned}
$$

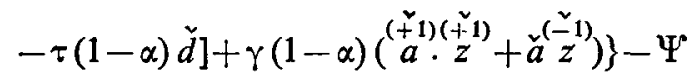

when $z_{0}=z_{N}=0,\left.z\right|_{t=0}=z^{0}$.

We shall first consider the problem for $\bar{z}$ corresponding to the initial function $\left|z^{0}\right|$, the right-hand part $|\Psi|$ and the zero boundary conditions.

Then, when $\gamma \leqslant c_{*} / 2(1-\alpha) k^{*}$, from the negativity of the right-hand part of (7) when $t=0$, it follows easily that $\bar{z}_{i}^{j} \geqslant 0$. Continuing the reasoning, we obtain $z_{i}^{-j} \geqslant 0$.

For (7) for $\bar{z}$ we have

and

$$
\|\bar{z}\|_{0} \leqslant(1+M \tau)\left(\|\check{z}\|_{0}+\|\Psi\|_{0}\right)
$$

$$
\|\bar{z}\|_{0} \leqslant M\left(\left\|z^{0}\right\|_{0}+\sum_{\omega_{\tau}^{t}}\|\Psi\|_{0} \tau\right) .
$$

Considering now the problem for $\overline{\bar{z}}=\bar{z}-z$ with the same limitation on $\gamma$, analogously to the foregoing we obtain

$$
\|\overline{\bar{z}}\|_{0} \leqslant M\left(\left\|z^{0}\right\|_{0}+\sum_{\omega_{\tau}^{t}}\|\Psi\|_{0} \tau\right) .
$$

Noting that $z=\bar{z}-\overline{\bar{z}}$, and bearing in mind the estimates for $\bar{z}$ and $\bar{z}$, we obtain the desired estimate (5).

From the principle of the maximum for the scheme

$$
\left(\begin{array}{l|l}
* * * & \alpha \\
* * \alpha & 1-\alpha
\end{array}\right)
$$

there follows uniform stability with respect to initial data and to the right-hand side. For the implicit scheme

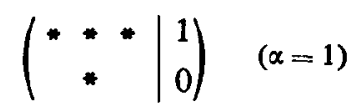

stability with respect to the norm \|\|$_{0}$ takes place at any $h$ and $\tau$. The requirement $d \geqslant 0$ is not a limitation, since with the aid of the transformations $z_{i}^{j}=s_{j} v_{i}^{j}$ and the corresponding choice of $s_{j}$ it is always possible to achieve satisfaction of the condition $d \geqslant 0$. 
2. Stability in the mean with respect to initial data. We shall deal with the question of the stability of difference schemes in the norm \|\|$_{2}$ with respect to initial data. For this purpose we shall obtain the integral identity

$$
\begin{aligned}
\frac{1+\beta}{2}\left(\rho^{(\alpha)}, z^{2}\right)_{t} & +\frac{\tau(1-\beta)}{2}\left(\rho^{(\alpha)}, z_{\bar{t}}^{2}\right)+0.5 I+((0.5-\alpha)(1+\beta)+0.5 \beta) \check{I}+ \\
& +(\alpha-0.5) \tau^{2}\left(a, z_{\bar{x} t}^{2}\right]+\left((2 \alpha-1+\alpha \beta) a+(1-\alpha) \check{a}, z_{\bar{x}} \check{z}_{\bar{x}}\right]- \\
& -\tau(\alpha-0.5)\left(a_{\bar{t}}, \check{z}_{\bar{x}}^{2}\right]=(z+\beta \check{z}, \Psi)+\frac{1+\beta}{2}\left(\rho_{\bar{t}}^{(\alpha)}, \check{z}^{2}\right)- \\
& -\alpha\left(d, z^{2}\right)-(1-\alpha) \beta\left(\check{d}, \check{z}^{2}\right)-(\alpha \beta d+(1-\alpha) \check{d}, z \check{z}),
\end{aligned}
$$

where $I=\left(a, z_{\bar{x}}^{2}\right],\left.\quad \rho^{(\alpha)}\right|_{t=0}=\left.\rho\right|_{t=0}, \quad z_{\bar{x}}^{2}=\left(z_{\bar{x}}\right)^{2}$.

We shall multiply equation (6) by $z+\beta \check{z}$; summing with respect to $\omega_{x}^{1}$ and using, by analogy with [8] the first Green's formula, in the case of the first boundary-value problem

$$
\begin{aligned}
\frac{1+\beta}{2}\left(\rho^{(\alpha)}, z^{2}\right) \bar{t} & +\frac{1+\beta}{2}\left(\rho_{\bar{t}}^{(\alpha)}, \check{z}^{2}\right)+\frac{\tau(1-\beta)}{2}\left(\rho^{(\alpha)}, z_{\bar{t}}^{2}\right)+\alpha I+ \\
& +(1-\alpha) \beta \ddot{I}+\left(\alpha_{i}^{2} a+(1-\alpha) \check{a}, z_{\bar{x}} \check{z}_{\bar{x}}\right]+\alpha\left(d, z^{2}\right)+ \\
& +(1-\alpha) \beta\left(\check{d}, \check{z}^{2}\right)+(\beta \alpha d+(1-\alpha) \check{d}, z \check{z})=(z+\beta \check{z}, \Psi) .
\end{aligned}
$$

From this, bearing in mind the transformations

$$
\begin{gathered}
\alpha I+(1-\alpha) \beta \check{I}=0.5 I+(\alpha-0.5)(I+\check{I})+((0.5-\alpha)(1+\beta)+0.5 \beta) \check{I}, \\
I+\check{I}-2\left(a, z_{x}^{-} \check{z}_{x} \mathbf{l}=\tau^{2}\left(a, z_{x t}^{2}\right]-\tau\left(a_{\grave{t}}^{-}, \check{z}_{x}^{2}\right],\right.
\end{gathered}
$$

we obtain the integral identity (8).

We shall consider the question of stability with respect to initial data for the case $\alpha=\frac{1}{2}$.

By putting in (8) $\beta=1, \alpha=\frac{1}{2}, \Psi=0$ we shall obtain

$$
\begin{gathered}
\left(\rho^{(\alpha)}, z^{2}\right) \bar{t}+0 \cdot 5(I+\check{I})+0 \cdot 5\left(a+\check{a}, z_{\bar{x}} \check{z}_{\bar{x}}\right] \\
=-\frac{1}{2}\left(d, z^{2}\right)-\frac{1}{2}\left(\check{d}, \check{z}^{2}\right)-\frac{1}{2}(d+\check{d}, z \check{z})+\left(\rho_{\check{t}}^{(\alpha)}, \check{z}^{2}\right),
\end{gathered}
$$

Using the estimate from [8]:

$$
I \leqslant\left. M\left(\rho^{(\alpha)}, z_{x}^{2}\right]\right|_{t=0}, \text { where }\left.\rho^{(\alpha)}\right|_{t=0}=\left.\rho\right|_{t=0},
$$

and using the inequalities

$$
\begin{gathered}
\frac{a+\check{a}}{2 \sqrt{\bar{a} \check{a}}} \leqslant 1+M \tau,\left(1, z^{2}\right) \leqslant M I, \\
-\left.M \delta^{2}\left(\rho^{(\alpha)}, z^{2}\right)\right|_{t=0} \leqslant-M \tau^{2}(I+\check{I}) \leqslant 0 \cdot 5(I+\check{I})+0 \cdot 5\left(a+\check{a}, z_{\check{x}} \check{z} \bar{x}\right],
\end{gathered}
$$

we obtain from (9)

$$
(1-M \tau) w \leqslant(1+M \tau) \check{w}+\tau M \delta^{2} w^{0},
$$

where $w=\left(\rho^{(\alpha)}, z^{2}\right), \delta=\varepsilon / h$. 
From this there follows

and

$$
w \leqslant M\left(1+\delta^{2}\right) w^{0}
$$

$$
\|z\|_{2} \leqslant M \sqrt{1+\delta^{2}}\left\|z^{0}\right\|_{2}
$$

From the preceding inequality, by virtue of [14] there follows.

THEOREM 1. If condition (S) is satisfied, then the scheme $\mathcal{P}_{h \tau}^{(\alpha)}$ when $\alpha=\frac{1}{2}$ is stable in the mean with respect to initial data for an arbitrary $\delta=\tau / h \rightarrow \infty$.

Note. Using the integral identity (8) when $\beta=0$ and $\Psi=0$, it is easy to obtain for $0 \leqslant \alpha<\frac{1}{2}, \frac{1}{2}<\alpha \leqslant 1$ the following estimates:

$$
\begin{aligned}
& \|z\|_{2} \leqslant \frac{M_{1}(\gamma) \mathrm{e}^{M / \varepsilon}}{\sqrt{\varepsilon}}\left\|z^{0}\right\|_{2}, \quad \gamma=\frac{\tau}{h^{2}} \leqslant \frac{c_{*}(1-\varepsilon)}{2(1-2 \alpha) k^{*}}, \quad 0 \leqslant \alpha<\frac{1}{2}, \\
& \|z\|_{2} \leqslant M_{2}(\gamma)\left\|z^{0}\right\|_{2} \text { for } \frac{1}{2}<\alpha \leqslant 1 \text { and any } \gamma,
\end{aligned}
$$

where $\varepsilon$ is any number from the interval $0<\varepsilon<1$ (in the case $\alpha=1$ the constant $M_{2}$ does not depend on $\gamma$ ).

There follows from this, in accordance with [14], stability with respect to the norm \|\|$_{2}$, stability in the mean with respect to initial data when $\alpha \neq \frac{1}{2}$ and at an arbitrary $\gamma \rightarrow \infty$ with satisfaction of condition (S).

If we reject the requirement to limit the difference ratios (e.g., in the case of an oblique discontinuity) $a_{\bar{t}}$ and $d_{\bar{t}}$ then, by using the method of integral inequalities, we can obtain the following estimate:

$$
\|z\|_{2} \leqslant M \sqrt{1+(1-\alpha)^{2} \gamma^{2}}\left\|z^{0}\right\|_{2}+M\left\{\sum_{\omega_{\tau}^{t}} \tau\|\Psi\|_{2}^{2}\right\}^{\frac{1}{2}} \quad(0.5 \leqslant \alpha \leqslant 0) .
$$

The estimate is obtained on the assumption of the limitation of $p_{\bar{t}}$.

3. A priori estimates and stability with respect to initial data and the right-hand side. In [8], to solve the problem

$$
\overline{\mathcal{P}}_{h \tau}^{(\alpha)} z=-\Psi, \quad z_{0}=z_{N}=0,\left.\quad z\right|_{t=0}=z^{0}, \quad 0.5 \leqslant \alpha \leqslant 1 .
$$

the following a priori estimates were derived:

$$
\begin{gathered}
\left\|z_{0}\right\| \leqslant\|z\|_{2}+\left\|z_{\bar{x}}^{-}\right\|_{2} \leqslant M\left\{\left\|z^{0}\right\|_{2}+\left\|z_{\bar{x}}^{0}\right\|_{2}+\left(\sum_{\omega_{\tau}^{t}}\|\Psi\|_{2}^{2} \tau\right)^{\frac{1}{2}}\right\}, \\
\left(1, z^{2^{m}}\right)^{\frac{1}{2 m}} \leqslant C_{m}\|\tilde{\Psi}\|_{3} . \\
\left(\sum_{\omega_{\tau}^{t}} z^{2^{m}} \tau\right)^{\frac{1}{2} m} \leqslant C_{m}\|\tilde{\Psi}\|_{3}, \quad \alpha=1, \quad z_{0}=0,
\end{gathered}
$$

where $C_{m}=M 2^{m} m e^{\mathrm{M}^{m_{m}}}$. Estimate (11) was obtained with the condition $\left|a_{t}\right| \leqslant M,\left|p_{t}\right| \leqslant M$, and (12) and (13) on the assumption that only $\left|p_{t}^{-}\right|$is bounded. Estimates (12) and (13) will be used to study convergence in the case of moving discontinuities.

Using formula (8) when $\beta=-1$, we can obtain, by a method analogous to [8], an $a$ priori estimate when $0 \leqslant \alpha \leqslant \frac{1}{2}, z^{0}=0$ and on condition (S): 


$$
\|z\|_{0} \leqslant \frac{M \mathrm{e}^{M / t}}{\sqrt{\varepsilon}}\left(\sum_{\omega_{\tau}^{*}}\|\Psi\|_{2}^{2} \tau\right)^{\frac{1}{t}} \text { when } \gamma \leqslant \frac{c_{*}(1-\varepsilon)}{2(1-2 \alpha) k^{*}}, \quad \varepsilon \in(0 \cdot 1) .
$$

From formulae (11) and (14) there follows the stability with respect to the right-hand side $\left(z^{0}=0\right)$ :

$$
\|z\|_{0} \leqslant M\left(\sum_{\omega_{\tau}^{t}}\|\Psi\|_{2}^{2} \tau\right)^{\frac{1}{2}} \leqslant M\left(\sum_{\omega_{\tau}^{t}}\|\Psi\|_{0}^{2} \tau\right)^{\frac{1}{t}},
$$

and also with respect to initial data $(\Psi=0)$ :

$$
\|z\|_{0} \leqslant\|z\|_{2}+\left\|z_{\bar{x}}\right\|_{2} \leqslant M\left\{\left\|z^{0}\right\|_{2}+\left\|z z_{x}^{0}\right\|_{2}\right\} .
$$

\section{§ 3. CONVERGENCE OF DIFFERENCE SCHEMES IN THE CLASS OF DISCONTINUOUS COEFFICIENTS}

In this section we shall prove a number of theorems concerning the convergence (and accuracy) of the solution of difference problem (II) to the solution of problem (I) when $h \rightarrow 0$ and $\tau \rightarrow 0$. Two cases are considered individually:

(a) moving discontinuities (the lines of discontinuity $k(x, t)$ are straight, $x=$ const.),

(b) oblique or moving discontinuities on the curves $x=\eta_{v}(t), v=1,2, \ldots, v_{0}$ and $\eta_{\nu}^{\prime}(t) \not \equiv 0$ at least for one $v$.

An especial part is played by the scheme $\mathscr{P}_{h \tau}^{(a)}$, which is defined by the standard functionals

$$
R[\psi(s)]=D[\psi(s)]=F[\psi(s)]=\int_{-0.5}^{0.5} \psi(s) \mathrm{d} s, \quad A[\psi(s)]=\left[\int_{-1}^{0} \frac{\mathrm{d} s}{\psi(s)}\right]^{-1},
$$

so that

$$
\begin{gathered}
a=\left[\int_{-1}^{0} \frac{\mathrm{d} s}{k(x+s h, t)}\right]^{-1}, \quad d=\int_{-0.5}^{0.5} q(x+s h, t) \mathrm{d} s, \quad \rho=\int_{-0.5}^{0.5} c(x+s h, t) \mathrm{d} s, \\
\Phi=\int_{-0.5}^{0.5} f(x+s h, t) \mathrm{d} s .
\end{gathered}
$$

Henceforward we shall call it scheme $(\Lambda)$

Let us formulate condition, which is to be used below. Condition $\mathrm{A}_{\alpha}$. The functions $k^{\prime}, k^{\prime \prime}, c^{\prime} q^{\prime}, f^{\prime}, u^{\prime \prime}\left(k u^{\prime}\right)^{\prime \prime}$ satisfy the Lipschits condition with respect to $x$, and the functions

$$
k, c, \partial^{\mathrm{m}_{\alpha}-1} c / \partial t^{m_{\alpha}-1}, \quad \partial^{m_{\alpha}} u / \partial t^{m_{\alpha}}
$$

the Lipschits condition with respect to $t\left(m_{\alpha}=1\right.$ when $\left.\alpha \neq \frac{1}{2}, m_{\frac{1}{2}}=2\right)$ either in the whole domain $\bar{\not}$ or in each of the subdomains $\Delta_{v}\left(v=0,1,2, \ldots, v_{0}\right)$ (henceforward this will always be indicated).

We shall consider first the question of convergence in the case of continuous coefficients. 
1. Convergence in the class of continuous coefficients.

THEOREM 2. If the conditions $\mathrm{A}_{\alpha}$ are satisfied in the domain $\overline{\bar{A}}$ then any scheme $\mathcal{P}_{\mathrm{h \tau}}^{(\alpha)}$ from the initial family of schemes has a second order of accuracy with respect to $h$ and an order $m_{\alpha}$ with respect to $\tau$, or more accurately, at a sufficiently small, $\tau \leqslant \tau_{0}$ the estimates
a) $\|y-u\|_{0} \leqslant M\left(h^{2}+\tau^{m} \alpha\right)$
$0.5 \leqslant \alpha \leqslant 1$,
b) $\|y-u\|_{0} \leqslant M\left(h^{2}+\tau\right)$, $\alpha=0$,

are satisfied, and in the case $\alpha=0$ the requirement,

$$
\gamma=\frac{\tau}{h^{2}} \leqslant \frac{c_{*}}{2 k^{*}}
$$

must be satisfied.

As follows from $\S 1$ (Section 3 and 4) for the right-hand side of problem (III) the relation

is satisfied.

$$
\|\Psi\|_{0}=O\left(h^{2}\right)+O\left(\tau^{m} \alpha\right)
$$

Using the a.priori estimate given in (8) (see $\S 2$, Section 3) and bearing in mind that $\|\Psi\|_{2} \leqslant\|\Psi\|_{0}$, we obtain estimate (a). Estimate (b) follows from the principle of the maximum ( $(2$, Section 1; see also (5)).

2. Error of approximation in the vicinity of the line of discontinuity. Let the function $k(x, t)$ and all the remaining coefficients of equation (1) have discontinuities of the first kind on a finite number of straight lines $x=\eta_{v}=x_{n_{v}}+\theta_{v} h\left(x_{n_{v}}\right.$ $\left.=n_{v} h, 0 \leqslant \theta_{\nu} \leqslant 1, \nu=1,2, \ldots, \nu_{0}\right)$ parallel to the axis $t$, the limit values on the left and right of the functions

$$
q, f, k^{\prime}, q^{\prime}, c^{\prime}, f, k^{\prime \prime}, u^{\prime}, u^{\prime \prime}, u^{\prime \prime \prime}
$$

satisfying along each line of discontinuity $x=\eta_{\nu}$ the Lipschits condition with respect to $t$ for $0 \leqslant t \leqslant T$ (condition $\mathrm{B}$ ).

If, moreover, in each of the domains $\Delta_{y}$, condition $A_{\alpha}$ is satisfied, then

$$
\Psi=O\left(h^{2}\right)+O\left(\tau^{m}\right)
$$

at all the points $(x, t)$ of the mesh $\Omega$ for which $x \neq x_{n_{y}}, x \neq x_{n_{y}+1}$.

To simplify the notation we shall deal with one line $\eta=x_{n}+\theta h, n=n(h)$, $\theta=\theta(h)$. Transition to the general case $v_{0}$ of lines of discontinuity does not present difficulty.

We find in [7] the expansion of $\varphi+\chi=\psi$ in powers of $h$ at the points $x=x_{n}$ and $x=x_{n+1}$. It turns out that for the scheme $(\Lambda)$ described in $\S 1$, Section 3 .

$$
\begin{gathered}
\varphi_{n}=O(1), \quad \varphi_{n+1}=O(1), \\
\varphi_{n}+\varphi_{n+1}=(0 \cdot 5-\theta)\left\{\left(L^{(k, q, f)} u\right)_{\mathrm{r}}-\left(L^{(k, q, f)} u\right)_{1}\right\}+O(h) .
\end{gathered}
$$

For any scheme $\mathcal{P}_{h \mathfrak{\tau}}^{(\alpha)}$ from the class of schemes under consideration the conditions 
are satisfied.

$$
h \varphi_{n}=O(1), \quad h \varphi_{n+1}=O(1), \quad \varphi_{n}+\varphi_{n+1}=O(1)
$$

We shall calculate $\chi_{n}$ and $\chi_{n+1}$, bearing in mind in the process that

$$
\left.\left[\frac{\partial u}{\partial t}\right]\right|_{\Gamma_{\nu}}=\left(\frac{\partial u}{\partial t}\right)_{\mathrm{r}}-\left(\frac{\partial u}{\partial t}\right)_{1}=0 .
$$

In the case of scheme $(\Lambda)$ we have

$$
\rho=\int_{-0.5}^{0.5} c(x+s h, t) \mathrm{d} s .
$$

Considering separately each of the cases $0 \leqslant \theta \leqslant 0.5$ and $0.5 \leqslant \theta \leqslant 1$ we find

$$
\begin{gathered}
\chi_{n}=O(1), \quad \chi_{n+1}=O(1), \\
\chi_{n}+\chi_{n+1}=-(0 \cdot 5-\theta)\left(c_{\mathrm{r}}-c_{1}\right) \frac{\partial u}{\partial t}(\eta, t)+O(h) .
\end{gathered}
$$

Combining these results with (15) and bearing in mind the equation $\mathcal{P} u=0$ and also the estimate

we obtain

$$
r_{n}=O\left(\tau^{m_{\alpha}}\right), \quad r_{n+1}=O\left(\tau^{m}\right)
$$

$$
(\varphi+\chi)_{n}+(\varphi+\chi)_{n+1}=(0 \cdot 5-\theta)\left\{(\mathcal{P} u)_{r}-(P \mathcal{P} u)_{1}\right\}+O(h)=O(h)
$$

and hence for the scheme $(\Lambda)$ we have

$$
\psi_{n}=O(1), \quad \psi_{n+1}=O(1), \quad \text { where } \psi=\varphi+\chi, \quad \psi_{n}+\psi_{n+1}=O(h) .
$$

For any scheme $\mathcal{P}_{h \tau}^{(\alpha)}$ the conditions

$$
h \psi_{n}=O(1), \quad h \psi_{n+1}=O(1), \quad \psi_{n}+\psi_{n+1}=O(1) .
$$

are satisfied.

The expressions for $\psi_{n}$ and $\psi_{n}+\psi_{n+1}$ may be represented in the form

$$
\psi_{n}=\frac{\beta_{(-1)}}{h}+\beta_{(0)}+h_{i(1)}+O\left(h^{2}\right), \quad \psi_{n}+\psi_{n+1}=\sigma_{(0)}+h \sigma_{(1)}+O\left(h^{2}\right)
$$

Without writing out the expressions for $\beta_{(-1)}, \beta_{(0)}, \beta_{(1)}, \sigma_{(0)}$ and $\sigma_{(1)}$ we shall indicate that $\beta_{(-1)}$ depends on $\theta, k_{l}, k_{r}\left(k_{l}, u_{l}^{\prime}=k_{r} u_{r}^{\prime}\right), \beta_{(0)}$ and $\sigma_{(0)}$ on $\theta, k_{l}, k_{r}, c_{l}, c_{r}, q_{l}, q_{r}$, $f_{l}, f_{r}, k_{l}^{\prime} k_{r}^{\prime}, u_{l}^{\prime}, u_{r}^{\prime}, u_{l}^{\prime \prime}, u_{r}^{\prime \prime}$ and the coefficients $\beta_{(1)}$ and $\sigma_{(1)}$ also depend on $k_{l}^{\prime \prime}, k_{r}^{\prime \prime}$, $q_{l}^{\prime}, q_{r}^{\prime}, f_{l}^{\prime}, f_{r}^{\prime}, c_{l}^{\prime}, c_{r}^{\prime}, u_{l}^{\prime \prime \prime}, u_{r}^{\prime \prime \prime}$, the relationship of the coefficients $\beta_{(-1)}, \beta_{(0)}, \beta_{(1)}, \sigma_{(0)}$ and $\sigma_{(1)}$ with respect to all arguments except $k_{l}$ and $k_{r}$ being linear.

For the scheme $(\Lambda)$ we have

$$
\beta_{(-1)}=0, \quad \sigma_{(0)}=0 .
$$

We shall represent $\psi=\varphi+\chi$ in the form of the sum

$$
\psi=\bar{\psi}+\overline{\bar{\psi}}
$$


where

$$
\begin{array}{ll}
\bar{\psi}=0 \text { when } \quad x \neq x_{n} \quad \text { and } x \neq x_{n+1}, \\
\bar{\psi}_{n}=\frac{\beta_{(-1)}}{h}+\beta_{(0)}+h_{r(1)}, & \bar{\psi}_{n}+\bar{\psi}_{n+1}=\sigma_{(0)}+h \sigma_{(1)} .
\end{array}
$$

From this and from (16) it is clear that

for all schemes $\mathcal{P}_{h \tau}^{(\alpha)}$.

$$
\|\overline{\bar{\psi}}\|=O\left(h^{2}\right)
$$

We shall represent the solution of problem (III) as the sum

$$
z=v+w,
$$

where $w$ is the solution of the "stationary" problem

$$
\left(a w_{\bar{x}}\right)_{x}=-\bar{\psi}, \quad w_{0}=0, \quad w_{N}=0 .
$$

The right-hand side $\Psi$ of equation (III) can be represented in the form

where

$$
\Psi=\alpha \psi+(1-\alpha) \check{\psi}+r=\bar{\Psi}+\bar{\Psi},
$$

$$
\bar{\Psi}=\alpha \bar{\psi}+(1-\alpha) \stackrel{\check{\psi}}{\dot{\psi}}, \quad \overline{\bar{\Psi}}=\alpha \overline{\bar{\psi}}+(1-\alpha) \overline{\bar{\psi}}+r .
$$

It follows from this that the function $v$ is defined by the conditions

where

$$
\left.\begin{array}{l}
\bar{P}_{h \tau}^{(\alpha)} v=-Q \quad \text { on } \quad \Omega, \\
v_{0}=v_{N}=0, \\
v^{0}=-w^{0},
\end{array}\right\}
$$

$$
Q=\overline{\bar{\Psi}}-p^{(\alpha)} w_{t}-\alpha d \cdot w-(1-\alpha) \check{d} \cdot \check{w} .
$$

3. Theorem concerning convergence in the case of stationary discontinuities. Let us turn to the estimation of $w$ and $w_{\bar{t}}$. Problem (IV) was investigated in (7), where it was shown that

$$
\|w\|_{0} \leqslant \bar{M}\|\psi\|_{4} \leqslant M h^{\alpha}
$$

here $x=2$ for scheme $(\Lambda), x=1$ for any scheme $\mathcal{P}_{\pi \tau}^{(\alpha)}$ from the class of schemes under consideration.

LEMMA 1. If the conditions B are satisfied, then for the solution of problem (IV) the following estimates are valid:
a) $\left\|w_{\vec{t}}\right\|_{0} \leqslant M h^{x}$,
b) $\left\|\boldsymbol{w}_{\boldsymbol{x}}\right\|_{2} \leqslant \boldsymbol{M} \boldsymbol{h}^{\boldsymbol{x}-\boldsymbol{t}}$.

In fact, taking into account that $\left(d_{w_{\bar{x}}}\right)_{\bar{t}}=a w_{\overline{x t}}+a_{\bar{t}} \check{w}_{\bar{x}}$, we obtain for $\zeta=w_{\bar{t}}$ the boundary-value problem

where

$$
\left(a_{\cdot}^{3} \cdot \bar{x}\right)_{x}=-\tilde{\psi}, \quad \zeta_{0}=\zeta_{N}=0,
$$

$$
\tilde{\psi}=\psi_{\dot{t}}+\left(a_{\bar{t}} \dot{w}_{\bar{x}}\right)_{x} .
$$


We now take advantage of the a priori estimate [7]

$$
\|\zeta\|_{0} \leqslant M\|\tilde{\tilde{\Psi}}\|_{4} \leqslant M\left\{\left\|\bar{\psi}_{\bar{t}}\right\|_{4}+\left\|\tilde{w}_{\bar{x}}\right\|_{1}\right\} .
$$

By virtue of condition $B$ the quantities

$$
\left(\sigma_{(0)}\right)_{\bar{t}}, \quad\left(\sigma_{(1)}\right)_{\bar{t}}, \quad\left(\beta_{(0)}\right)_{\bar{t}}, \cdot\left(\beta_{(-1)}\right)_{\bar{t}}
$$

are bounded in absolute magnitude, therefore

$$
\left\|\bar{\psi}_{\bar{t}}\right\|_{4} \leqslant M h^{x} \text {. }
$$

Introducing the Green's difference function of problem (IV) and representing $w$ in the form

$$
w=(G, \bar{\psi})
$$

it is not difficult to see that

$$
w_{x}=O\left(h^{x}\right) \quad \text { when } \quad x \neq x_{n+1}, \quad\left(w_{\bar{x}}\right)_{n+1}=O\left(h^{x-1}\right) .
$$

From this there immediately follows

The lemma is this proved.

$$
\left\|\check{w}_{\bar{x}}\right\|_{1}=O\left(h^{\varkappa}\right), \quad\left\|\hat{w}_{\bar{x}}\right\|_{2}=O\left(h^{\alpha-1}\right) .
$$

THEOREM 3. If condition $\mathrm{B}$ and conditions $\mathrm{A}_{\alpha}$ are satisfied in each of the domains $\Delta_{v}\left(v=0,1, \ldots, v_{0}\right)$ then the solution of problem (II) converges to the solution of problem (I) with the independent approach of $h$ and $\tau$ to zero, so that at a sufficiently small $\tau \leqslant \tau_{0}$ for any scheme $\mathscr{P}_{h \tau}^{(\alpha)}$ from the class of schemes under consideration the estimates

$$
\begin{array}{ll}
\|y-u\|_{a} \leqslant M(h+\tau) & \text { for } \alpha=1, \\
\|y-u\|_{0} \leqslant M\left(h^{\frac{1}{2}}+\tau^{m_{\alpha}}\right) & \text { for } 0.5 \leqslant \alpha<1 .
\end{array}
$$

are valid, where $m_{\alpha}=1$ when $\alpha \neq \frac{1}{2}, m_{\mathrm{z}}=2$.

THEOREM 4. If the conditions of Theorem 3 are satisfied, then for scheme $(\Lambda)$ the estimates

are valid.

$$
\begin{array}{ll}
\|y-u\|_{0} \leqslant M\left(h^{2}+\tau\right) & \text { for } \alpha=1, \\
\|y-u\|_{0} \leqslant M\left(h^{3 / x}+\tau^{m} \alpha\right) & \text { for } 0.5 \leqslant \alpha<1 .
\end{array}
$$

Note. For all $0 \leqslant \alpha \leqslant 1$ with the additional conditions

the estimate

$$
\gamma=\frac{\tau}{h^{2}} \leqslant \frac{c_{*}}{2(1-\alpha) k^{*}}
$$

is valid.

$$
\|y-u\|_{0} \leqslant M\left(h^{x}+\tau^{m} \alpha\right)
$$

Theorems 3 and 4 will be proved if we establish that estimates (19)-(22) and (24) are satisfied for the function $v$-the solution of problem (V), since

$$
\|z\|_{0} \leqslant\|v\|_{0}+\|w\|_{0} \leqslant M h^{x}+\|v\|_{0} .
$$


For the estimate of the solution of problem (V) in the case $0.5 \leqslant \alpha<1$ we shall use a priori estimate (11):

$$
\|v\|_{0} \leqslant M\left\{\left\|w_{\bar{x}}^{0}\right\|_{2}+\left(\sum_{\omega_{\tau}^{t}}\|Q\|_{2}^{2} \tau\right)^{\frac{1}{t}}\right\} .
$$

By virtue of [17] and Lemma 1 we have

$$
\|Q\|_{2} \leqslant M\left(h^{\alpha}+\tau^{\left.m_{\alpha}\right)}, \quad\left\|w_{x}^{0}\right\|_{2} \leqslant M h^{x-1} .\right.
$$

From this and from (25) there follow inequalities (20) and (22).

If condition (23) is satisfied, then to obtain estimates (19), (21) and (24) we can use the principle of the maximum:

since

$$
\|v\|_{0} \leqslant M\left\{\left\|v^{0}\right\|_{0}+\sum_{\omega_{\tau}^{t}} \tau\|Q\|_{0}\right\} \leqslant M\left(h^{\alpha}+\tau^{m} \alpha\right),
$$

$$
\left\|v^{0}\right\|_{0}=\left\|w^{0}\right\|_{0}=O\left(h^{\star}\right)
$$

The theorem is thus proved.

It must be borne in mind here that the $a$ priori estimate (11) applies only if $\left|a_{\bar{t}}\right|$ and $\left|\rho_{i}\right|$ are bounded (i.e., $\left|k_{i}\right| \leqslant M,\left|c_{i}\right| \leqslant M$ ). In the case of the moving discontinuity under consideration these conditions are satisfied.

4. Convergence in the case of an oblique discontinuity. We now consider the case of an "oblique discontinuity", i.e., the problem formulated in $\S 1$, Section 1 .

The coefficient $k(x, t)$ has discontinuities on a finite number of curves $\Gamma_{\nu}(v$ $\left.1,2, \ldots, v_{0}\right)$ satisfying the following conditions:

(1) the curves $\Gamma_{v}\left(v=0,1, \ldots, v_{0}+1\right)$ do not intersect in pairs;

(2) the curves $\Gamma_{v}$ are differentiable.

The coefficients $q(x, t)$ and $f(x, t)$ are also discontinuous only on the curves $\Gamma_{v}$, while $c(x, t)$ can have discontinuities only on these curves $\Gamma_{v}$, which are parallel to the axis $t\left(\eta_{v}^{\prime}(l) \equiv 0\right)$. All these conditions relative to the coefficients and the curves $\Gamma_{v}$ will be called the conditions $\mathrm{K}$.

TIIEOREM 5. If the conditions $\mathrm{K}$ and $\mathrm{A}_{\alpha}$ are satisfied in each of the domains $\Delta_{v}(v$ $=0,1, \ldots, v_{0}$ ) the solution of problem (II) when $\alpha=1$ converges to the solution of problem (I) with independent approach of $h$ and $\tau$ to zero, so that at sufficiently small $h \leqslant h_{0}$ and $\tau \leqslant \tau_{0}$

$$
\|y-u\|_{0} \leqslant M\left(h^{1-\rho_{2}(h)}+\tau^{1-\rho_{3}(t)}\right),
$$

where $p_{1}(h) \rightarrow 0$ when $h \rightarrow 0, p_{2}(\tau) \rightarrow 0$ when $\tau \rightarrow 0$, in the entire class of initial schemes.

THEOREM 6. If the conditions of Theorem 5 are satisfied, then for the scheme $(\Lambda)$ of the estimate

is valid.

$$
\|y-u\|_{0} \leqslant M\left(h^{1-\rho_{1}(h)}+\tau^{1-\rho_{3}(\tau)}\right) \quad \text { when } \alpha=1 .
$$

We shall prove both theorems for the case of one discontinuity:

$$
\eta=x_{n}+\theta h, \quad x_{n}=n h, \quad 0 \leqslant \theta \leqslant 1,
$$


where

$$
n=n(h, t), \quad \theta=\theta(h, t) .
$$

Let us consider the error

$$
\begin{array}{cc}
\Psi=\psi+r, \quad \psi=\varphi+\chi, & \varphi=L_{h}^{(k, q, f)} u-L^{(k, q, f)} u, \\
\chi=(c-p) \frac{\partial u}{\partial t}, \quad r=\rho\left(\frac{\partial u}{\partial t}-u_{t}\right) .
\end{array}
$$

If the conditions $\mathrm{A}_{\alpha}$ are satisfied in $\Delta v$, then $\psi=O\left(h^{2}\right)$ for $x \neq x_{n}, x \neq x_{n+1}$. Since $c(x, t)$ does not have a discontinuity on the line $\Gamma_{v}$ (when $\left.\eta_{v}^{\prime}(t) \not \equiv 0\right)$ then $\chi_{n}=0\left(h^{2}\right)$, $\chi_{n+1}=O(h)^{2}$.

For $\varphi_{n}$ and $\varphi_{n+1}$ we obtain the same expressions as in Section 2 but in this case

$$
\Psi_{n}+\Psi_{n+1}=O(1)
$$

even for scheme $(\Lambda)$, since on the curve $\Gamma_{v}\left(\eta_{v}(t) \neq 0\right)$ the derivative $\partial u / \partial t$ is discontinuous, which follows from the identity

$$
\left.\left[\frac{\partial u}{\partial t}\right]\right|_{\Gamma_{\nu}}=-\left.\eta^{\prime}(t)\left[\frac{\partial u}{\partial x}\right]\right|_{\Gamma_{v}} .
$$

In fact, for scheme $(\Lambda)$ we have

$$
\varphi_{n}+\varphi_{n+1}=(0.5-\theta) c(\eta(t), t)\left[\frac{\partial u}{\partial t}\right]+O(h) .
$$

Let us deal in detail with the estimation of the error $r$. The curve $x=\eta(t)$ for $t-\tau \leqslant t^{\prime} \leqslant t$ intersects the straight lines $x=i h$ at the points $\left(x_{p}, t_{p}^{\prime}\right)$ where $p=p_{0}, p_{0}+1, \ldots, p_{0}+\bar{n}-1$. At all these points $r_{p}=0(1)$. Let $\eta^{\prime}(t)>0$. Then

and hence

$$
\left(u_{\bar{t}}\right)_{p}=(1-\lambda)\left(\frac{\partial u}{\partial t}\right)_{r}+\lambda\left(\frac{\partial u}{\partial t}\right)_{2}+O(\tau)
$$

$$
r_{p}=\left.(1-\lambda) c_{p}\left[\frac{\partial u}{\partial t}\right]\right|_{\Gamma_{\vee}}+O(\tau)
$$

where $\lambda$ is determined from the condition

so that

$$
x_{p}=\eta(t-\lambda \tau), \quad 0 \leqslant \lambda \leqslant 1
$$

$$
\lambda \tau=\eta^{\prime}(t) \theta h+O(\tau h)
$$

Let us calculate the number of intersection $\bar{n}$. Three cases are possible (see Figure)
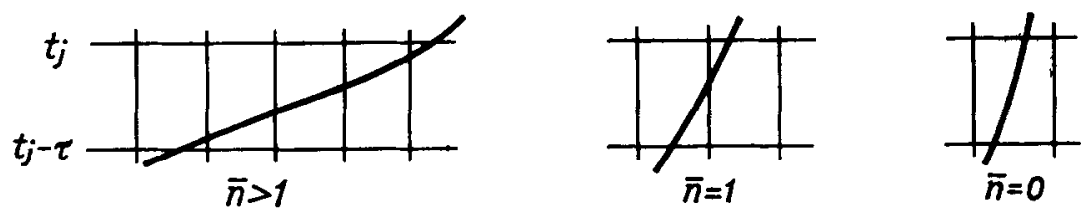
The number of intersections $\vec{n}$ on each line of the mesh satisfies the condition

$$
\bar{n} \leqslant \frac{|\eta-\check{\eta}|}{h}+1=\frac{|\eta-\check{\eta}|}{\tau} \frac{\tau}{h}+1 \leqslant M \frac{\tau}{h}+1 .
$$

LeMma 2. Let $\bar{v}$ be the solution of the problem

$$
\overline{\mathcal{P}}_{h \tau}^{(1)} \bar{v}=-\bar{r} \text { on } \Omega ; \quad \bar{v}_{0}=\bar{v}_{N}=0, \quad \bar{v}^{0}=0,
$$

where $\bar{r}=O(1)$ at one point $\left(x_{p}, t\right)$ on each line and $\bar{r}=0$ at all the remaining points of the mesh $\Omega$. Then for $\bar{v}$ when $h \leqslant h_{0}$ there is a valid estimate

where $\rho(h) \rightarrow 0$ when $h \rightarrow 0$.

$$
\|v\|_{0} \leqslant M h^{1-\rho(h)} \text {, }
$$

We shall use a priori estimate (12). From this estimate there follows the inequality

where

$$
\|\bar{v}\|_{0} \leqslant C_{m}\|\tilde{\bar{r}}\|_{3} h^{-1 m}, \quad m=1,2,3, \ldots,
$$

$$
C_{m}=M 2^{m} m \mathrm{e}^{M 2^{m} m} .
$$

Our calculations give us

and

$$
\|\bar{r}\|_{3}=h\left|\bar{r}_{p}\right| \leqslant M h
$$

$$
\|\bar{v}\|_{0} \leqslant C_{m} h^{1-1^{m}}
$$

We shall select the number $m$ dependent on $h$ so as to obtain estimate (26). For this we must have

$$
\rho(h) \geqslant \frac{1}{2^{m}}+\frac{M 4^{m}}{\ln (1 / h)} .
$$

Selecting, for example

we obtain

$$
2^{m} \sim \sqrt[3]{\ln (1 / h)}
$$

$$
\rho(h) \sim \frac{1}{\sqrt[3]{\ln (1 / h)}} .
$$

LEMMA 3. If the function $\vec{r}$ satisfies the conditions $\bar{h} r_{n}=O\left(h^{x-1}\right), h \bar{r}_{n+1}=O\left(h^{x-1}\right)$, $\bar{r}_{n}+\bar{r}_{n+1}=O(1)$ and $\bar{r}=0$ when $x \neq x_{n}, x \neq x_{n+1}(h=n(h, t))$ then for the solution of problem (VI) when $h \leqslant h$ there is valid the estimate

$$
\|\bar{v}\|_{0} \leqslant M h^{\alpha / 2-\rho(h)}
$$

where $p(h) \rightarrow 0$ when $h \rightarrow 0$.

For proof of the lemma it is sufficient to convince ourselves that

i.e.,

$$
\|\bar{r}\|_{2}^{2} \leqslant h^{3} r_{n}^{2}+h^{2}\left(\bar{r}_{n}+\bar{r}_{n+1}\right)^{2} \leqslant M h^{\alpha},
$$

$$
\|\bar{r}\|_{3} \leqslant M h^{\alpha / 2},
$$

and then use a priori estimate (12). 
LeMma 4. Let $\bar{v}$ be a solution of problem (VI), where $\bar{r}=O(1)$ at the points $p_{0}+1$, $p_{0}+2, \ldots, p_{0}+\bar{n}$ of the line $t=\tau j$ and $\bar{r}=O(\tau)$ at the remaining points of the line $t=\tau j$, the number of points $\bar{n}$ satisfying the condition

$$
0 \leqslant \bar{n} \leqslant M \frac{\tau}{h} .
$$

Then for $\bar{v}$ when $\tau \leqslant \tau_{0}$ there takes place the estimate

$$
\|\bar{v}\|_{0} \leqslant M \tau^{1-\rho(\tau)}, \quad \rho(\tau) \rightarrow 0 \quad \text { when } \tau \rightarrow 0 .
$$

For proof it is sufficient to estimate

$$
\|\bar{r}\|_{3} \leqslant M\left[\frac{\tau}{h}(M h)+\tau\right] \leqslant M \tau
$$

and use a priori estimate (13), from which it follows that

$$
\|\bar{v}\|_{0} \leqslant C_{m} \tau^{1-1-1 m} \text {. }
$$

Now, selecting $m$ dependent on $\tau$, we arrive, at sufficiently small values of $\tau$, at estimate (27).

Corollary. If in Lemma $4 \bar{n} \leqslant M \tau / h+1$ then by virtue of Lemmas 2 and 4 we will have $\|\bar{v}\|_{0} \leqslant M\left(h^{1-p_{1}(h)}+\tau^{1-p_{9}(t)}\right.$ when $\tau \leqslant \tau_{0}$ and $h \leqslant h_{0}$.

We shall now turn to the proof of Theorems 5 and 6 .

We shall represent solution $z$ in the form of the sum

$$
z=\bar{z}+\overline{\bar{z}}+v \text {, }
$$

where $v$ is the solution of problem (III) with a right-hand side equal to $r=p(\partial u / \partial t$ $\left.-u_{\bar{r}}\right)$, and $\bar{z}$ is the solution of the same problem, with the right-hand side

$$
\bar{\Psi}_{i}=\bar{\psi}_{n} \delta_{i n}+\bar{\psi}_{n+1} \delta_{i, n+1}, \quad \delta_{i k}= \begin{cases}1, & i=k, \\ 0, & i \neq k .\end{cases}
$$

An estimate for $\overline{\bar{z}}$ can be obtained with the aid of the maximum principle:

$$
\|\tilde{\tilde{z}}\|_{0} \leqslant M\left(h^{2}+\tau\right)
$$

for $v$ with the aid of the corollary of Lemmas 2 and 4, and for $z$ with the aid of Lemma 3.

Collating all the results, and noting that

$$
\|z\|_{0} \leqslant\|\bar{z}\|_{0}+\|\overline{\bar{z}}\|_{0}+\|v\|_{0},
$$

we obtain the desired estimates.

Note. From estimate $\left(10^{\prime}\right)$ there follows the convergence in the mean for the six-point scheme $(\Lambda)(0.5 \leqslant \alpha \leqslant 1)$ in the case of an oblique discontinuity.

In conclusion, the authors take this opportunity to express their gratitude to A. N. Tikhonov for discussing the results of this paper. 


\section{REFERENCES}

1. LEES, M., J. Soc. Industr. Appl. Math. 7: No. 2, 167-183, 1959.

2. LEES, M., Duke Math J. 27: No. 3, 297-311, 1960.

3. LEES, M., Trans. Amer. Math. Soc. 94: 58-73, 1960.

4. DOUGLAS, J., Trans. Amer. Math. Soc. 89: No, 2, 484-518, 1958.

5. KELLER, H., In: Math. methods for digital computers. New York-London, 135-143, 1960.

6. KAMYNIN, L. I., Dok1. Akad. Nauk SSSR 136: No. 6, 1277-1290, 1961.

7. TIKHONOV, A. N. and SAMARSKII, A. A., Zh. vych. mat. 1: No. 1, 5-63, 1961.

8. SAMARSKII, A. A., Zh. vych. mat. 1: No. 3, 441-460, 1961.

9. TIKHONOV, A. N. and SAMARSKII, A. A.; Dokl. Akad. Nauk SSSR 131: No. 3, 514-517, 1960.

10. TIKHONOV, A. N. and SAMARSKII, A. A., Dokl. Akad. Nauk SSSR 124: No. 4, 779-782, 1959.

11. SAMARSKI, A. A., Trud. Vses. soveshch. po differentsial'nym uravneniyam. (Proc. AllUnion Conf. on Differential Equations.) (Erevan, November, 1958) Erevan, Izd. Akad. Nauk Armenian SSSR 148-160, 1960.

12. SAMARSKII, A. A., Nauch. dok1. vysshei shkoly fiz.-mat. nauk (Scientific Papers of the Higher School. Physico-Maths. Sciences) 1959.

13. SAMARSKII, A. A., Dokl. Akad. Nauk SSSR 121: No. 2, 225-228, 1958.

14. RYABEN'KII, V.S., and FILIPPOV A. F., Ob ustoichivosti raznostnykh uravnenii. (The Stability of Difference Equations.) Gostekhizdat, Moscow-Leningrad, 1956.

15. RICHTMAYER R. D., Difference Methods of Solving Boundary Value Problems.

16. SAUL'EV, V. K., Integrirovanie uravnenii parabolicheskogo tipa metodom setok. (Integration of parabolic equations by the method of meshes.) Fizmatgiz, Moscow-Leningrad, 1960. 